\title{
Design of scaffolds with computer assistance
}

\author{
H. A. Almeida ${ }^{1}$, P. J. Bártolo ${ }^{1}$ \& J. C. Ferreira ${ }^{2}$ \\ ${ }^{1}$ Centre for Rapid and Sustainable Product Development - CDRsp, \\ Polytechnic Institute of Leiria, Portugal \\ ${ }^{2}$ Superior Institute of Technology, Technical University of Lisbon, \\ Portugal
}

\begin{abstract}
Advanced rapid prototyping techniques are being used to fabricate scaffolds for tissue engineering with controlled architecture. These techniques combine computer-aided design with bio-manufacturing tools to produce 3D structures in a multitude of materials. These scaffolds must be biocompatible, biodegradable, with appropriate porosity, pore structure and distribution, having both surface and structural compatibility. Structural compatibility of the host tissue corresponds to an optimal adaptation to the scaffold mechanical behaviour, referring to its mechanical properties and deformation capability. This paper proposes a computer tool to predict the mechanical behaviour of scaffolds with different topological architectures and levels of porosity. The influence of the interaction between biofluids and the scaffold is also presented.

Keywords: scaffolds, mechanical behaviour, tissue engineering and biofluid mechanics.
\end{abstract}

\section{Introduction}

The loss or failure of an organ or tissue is a frequent, devastating and costly problem in health care. Currently, this loss or organ failure is treated by either transplanting organs from one individual to another or performing surgical reconstructions by transferring the tissue from one location in the human body to the diseased site. The need for substitutes to replace or repair tissues or organs due to disease, trauma, or congenital problems is overwhelming. In 2003, in the USA alone, 87717 patients were waiting for organ transplantation [1]. This 
number has increased to 88598 by May 2005, while 4375 transplants were performed between January and February 2005 [2], leaving 84223 patients in the waiting list. Although clinics have tried to replace the function of failing organs mechanically or through implantation of synthetic replacements, these are often temporary solutions, not allowing the patient to completely resume normal activities. Infection and device rejection are serious secondary affects that may arise during such procedures causing in some cases very harmful setbacks. By the end of 2004, there were 153245 persons living with a functioning organ transplant in the United States. This number reflects an increase by about $1.8 \%$ over the prior year and a 1.7-fold increase since 1996 .

To overcome this organ deficiency, a new field called Tissue Engineering has been growing and gaining more and more importance in the scientific community. Tissue engineering represents a new, emerging and interdisciplinary field involving combined efforts of biologists, engineers, material scientists and mathematicians towards the development of biological substitutes to restore, maintain, or improve tissue functions [3].

In tissue engineering, most strategies has focussed on using biomaterials as scaffolds to direct specific cell types in organising three-dimensional structures and performing differentiated functions with the ultimate goal of replacing or restoring physiological functions lost in diseased or damaged organs [4].

These scaffolds are often critical, both ex vivo and in vivo, as they serve the following purposes [5,6]: allow cell attachment, proliferation and differentiation; deliver and retain cells and growth factors; enable diffusion of cell nutrients and oxygen, on top of enabling appropriate mechanical and biological environments for organised tissue regeneration. An ideal scaffold must satisfy some biological and mechanical requirements to achieve these goals [7].

These biological requirements are: biocompatibility (the scaffold material must be non-toxic and allow cell attachment, proliferation and differentiation), biodegradability (the scaffold material must degrade into non-toxic products), controlled degradation rate (the degradation rate of the scaffold must be adjustable in order to match the rate of tissue regeneration) and appropriate macro and microstructure of the pores and shape to allow tissue in-growth and vascularisation. The mechanical and physical requirements are: sufficient strength and stiffness to withstand stresses in the host tissue environment, adequate surface finish to guarantee that a good biomechanical coupling is achieved between the scaffold and the tissue, as well easy sterilisation by either exposure to high temperatures or immersing into a sterilisation agent, remaining unaffected by either of these processes.

The possibility to predict effective mechanical properties for tissue scaffolds is very important for tissue engineering applications. This paper introduces a computer tool that is being developed to support computer-aided design of rapid prototyping scaffolds for tissue engineering. This tool is particularly important as it enables to quantify the structural heterogeneity and the mechanical properties of a scaffold with a designed microstructure. 


\section{Computer-aided design of scaffolds for tissue engineering (CADS)}

The software CADS (Computer-Aided Design of Scaffolds) makes the bridge between different computer applications by managing database tools, CAD modelling systems and simulation tools based on the Finite Element Method. The main computer tools used by $C A D S$ are (Fig. 1):

- $\quad$ SOLIDWORKS for the 3D modelling of scaffolds.

- ABAQUS for numerical simulations.

- ANSYS for topological optimisation.

- ACCESS for the definition of both materials and simulations data bases.

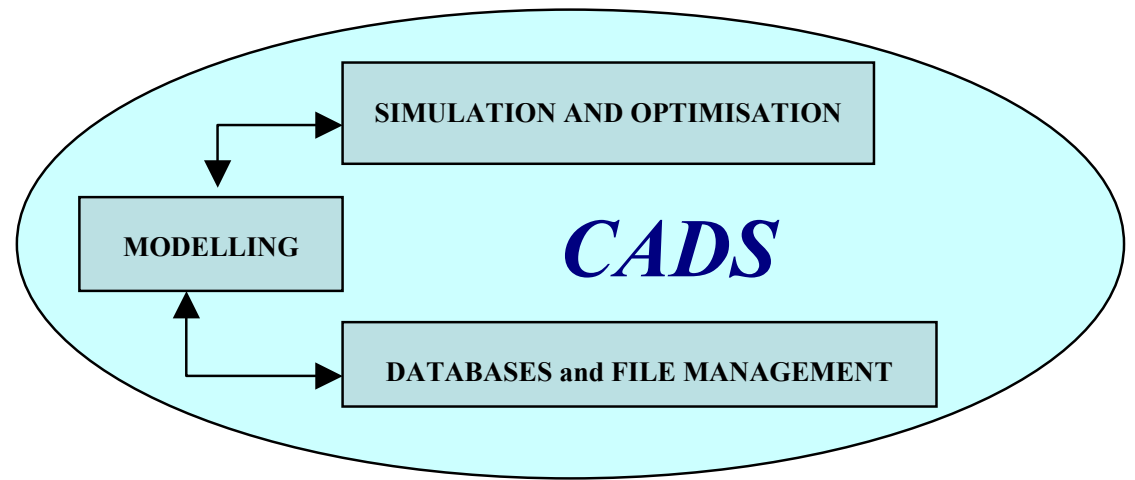

Figure 1: The different software tools managed by CADS.

The software $C A D S$ was developed by using a Delphi programming language and comprises four main areas:

- Evaluation of Scaffold's Porosity

- Evaluation of Scaffold's Modulus

- Material Database

- FEA Simulations.

These two first domains enable to obtain porosity evaluation as a function of a specific mechanical property (elastic and shear modulus) and the mechanical properties as a function of porosity, for a pre-defined material and scaffold topology. The evaluation is performed based on mathematical models obtained through computer simulation. The scaffold is considered as a LEGO structure formed by the association of small elementary elements or blocks.

Three types of elementary building blocks were considered as shown in Figures 2 and 3. Figure 2 illustrates elementary scaffolds of the " $n$ face pore blocks" family and Fig. 3 illustrates elementary scaffolds of the " $m$ pores per face blocks" family with circular pores. The " $m$ pores per face blocks" are 
scaffolds consisting of multiple pores in each unit, while maintaining specific scaffold porosity, ranging from 1 to 100 pores, with two types of topological architecture, circular and 4 face pores. All elementary family scaffolds are cubic scaffold units of $5 \mathrm{~mm}$ in size.

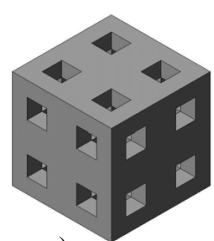

a)

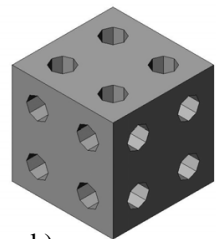

b)

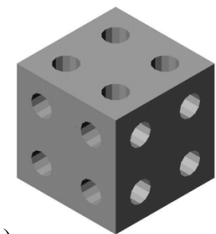

c)

d)

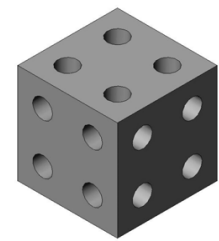

Figure 2: $\quad$ The $n$ face pore blocks family classified according to the number of faces per pore. a) 4 face (4f) pore unit, b) $8 \mathrm{f}$ unit, c) $12 \mathrm{f}$ unit and d) circular unit.

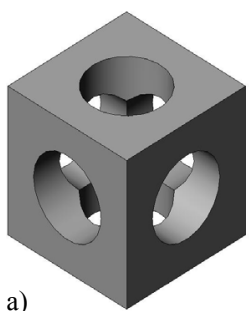

a)

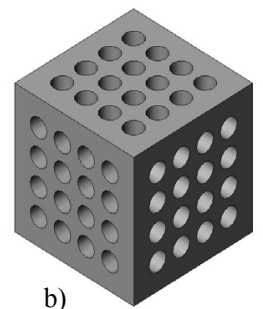

b)

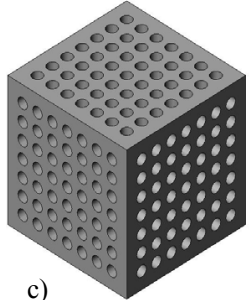

c)

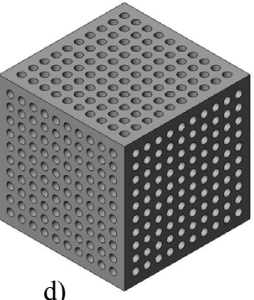

d)

Figure 3: The $m$ pores per face blocks family classified according to the number of pores per face. Only 4 of the 10 geometries with circular pores are represented. a) 1 pore (1p) unit, b) $16 \mathrm{p}$ unit, c) $49 \mathrm{p}$ unit and d) 100p unit.

The last two areas of CADS will enable to access and upgrade the database of material properties, as well to run both mechanical analysis simulations and topological optimisation. Data are available for a wide rage of materials for both hard and soft tissue applications (alginate, collagen, poly(glycolic) acid, poly(lactic) acid, hydroxyapatite). Hard tissues are usually stiffer (with higher elastic modulus) and stronger (with higher tensile strength) than soft tissues.

The influence of the scaffold's geometry within the normal flow of biofluids in the human body is another fundamental issue, so there is a need to perform Computer Fluid Dynamics simulations. These types of simulations are specially indicated to analyse the flow of fluids, either for gases or liquids. The first step is to obtain the geometry that represents the existing fluid. Taking into account $n$ face geometries, the empty space within the scaffold unit represents the volume of the biofluid, if the scaffold is hosted in a biofluid rich environment, see Fig. 4. Figure 5 illustrates the unfilled volumes of the scaffold units, which represents the volume of the biofluid. Presently, the biofluid is blood and the fluid geometries were obtained from $n$ face scaffold units with $50 \%$ porosity. 
a)

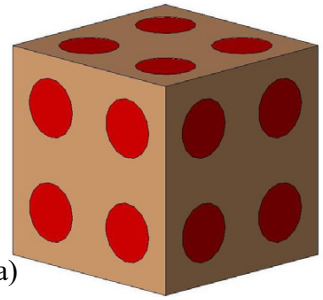

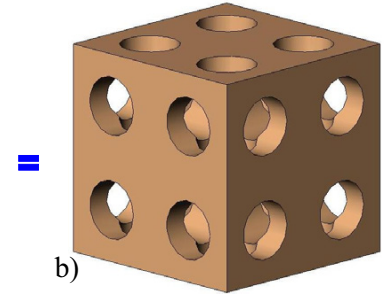

Figure 4: $\quad$ Illustration of a scaffold hosted within a biofluid environment and the resulting volumes. a) Scaffold with biofluid b) scaffold and c) biofluid geometry.

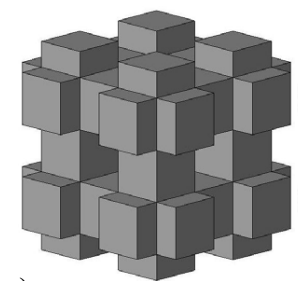

a)

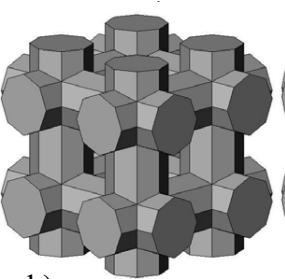

b)

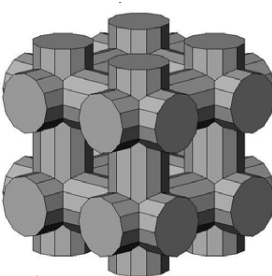

c)

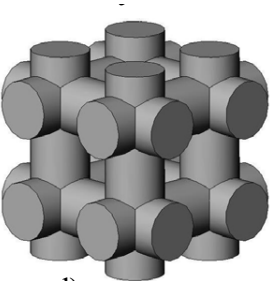

d)

Figure 5: The family of scaffolds representing the volume related with the flow of biofluids inside the human body, which is classified according to the number of faces per pore. a) 4 face (4f) pore unit, b) $8 \mathrm{f}$ unit, c) $12 \mathrm{f}$ unit and d) circular unit.

\section{Mechanical simulations}

To study the mechanical behaviour of scaffolds two different conditions were considered:

- A linear elastic behaviour for hard tissue applications,

- A hyperelastic behaviour for soft tissues, described through a polynomial form according to the Neo-Hookean model.

The main purpose of simulating scaffold mechanical behaviour is to evaluate the porosity dependence of both the elastic modulus and the shear modulus. For a given unit block with a specific open pore architecture, boundary and loading conditions used for calculating the mechanical properties are shown in Fig. 5. For the numerical calculation of the elastic modulus, a uniform displacement in a single direction is considered (the $\mathrm{X}$ direction), which is equivalent to the strain on the same direction (Ex), imposed to a face of the block (Face A). The opposite face (Face B) of the scaffold unit is constrained and unable to have any displacement. The average reaction force produced on Face $\mathrm{B}$ is used to determine the elastic modulus, due to the imposed displacement. 
For the numerical evaluation of the shear modulus, a uniform displacement is applied on the top of a surface, being the opposite face also unable to have any displacement. In a first approximation, each unit is considered isotropic.

a)

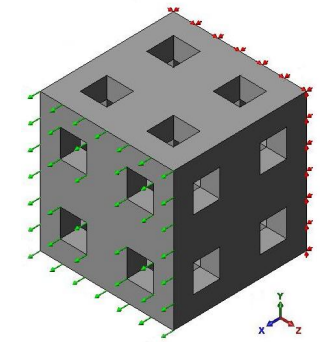

b)

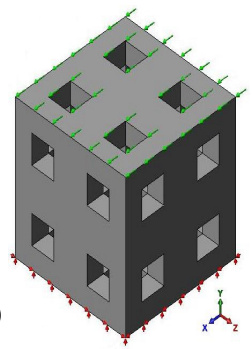

Figure 6: Loads and constraints for the numerical analysis. a) Tensile Solicitation and b) Shear Solicitation.

It is possible, through computer simulation, to establish a cubic mathematical law relating the scaffold porosity and the tensile modulus, as follows:

$$
\begin{gathered}
\mathrm{E}(\phi)=\mathrm{E}_{0}+\mathrm{C}_{1} \phi+\mathrm{C}_{2} \phi^{2}+\mathrm{C}_{3} \phi^{3} \\
\mathrm{G}(\phi)=\mathrm{G}_{0}+\mathrm{C}_{4} \phi+\mathrm{C}_{5} \phi^{2}+\mathrm{C}_{6} \phi^{3}
\end{gathered}
$$

where $\mathrm{E}$ corresponds to the scaffold elastic modulus, $\mathrm{E}_{0}$ corresponds to the material elastic modulus, $G$ corresponds to the scaffold shear modulus, $G_{0}$ corresponds to the material shear modulus, $\phi$ corresponds to the scaffold porosity and $\mathrm{C}_{1}, \mathrm{C}_{2} \ldots \mathrm{C}_{6}$ are material dependent constants.

Numerical simulations performed on the " $m$ pores per face blocks" are similar, intending to analyze the influence of the number of pores per face regarding the scaffold's mechanical behaviour. Keeping up a scaffold's porosity of $50 \%, 10$ unit blocks were designed with $m$ pores per face, ranging from 1 to 100 pores.

The effect of the pore architecture is illustrated by Fig. 7.a and 7.b, which show the decrease of both the elastic modulus and the shear modulus for Poly(caprolactone) with the increase of porosity. For tensile solicitations the findings show that a $4 \mathrm{f}$ unit is the unit with the worst performance below $50 \%$ porosity, becoming the best scaffold unit for higher percentages of porosity. Below 50\% porosity, the circular pore scaffolds are the units with better material modulus. For shear solicitations, the results show that the $4 \mathrm{f}$ unit is the unit with the worst performance, being the circular pore scaffolds the unit with better material modulus performance.

The influence of the deformation value on the mechanical behaviour of scaffolds is significant as expected. This can be observed at Fig. 8, which indicates the variation of the elastic modulus of circular pore scaffold units of alginate submitted to different deformation values. The material modulus decreases as the deformation increases. 
Another aspect of extreme importance is the number of pores per face. As illustrated in Fig. 9.a and 9.b, it is possible to verify that the scaffolds modulus increases with the number of pores per face for each unit. For the elastic modulus, the increase has a more stable variation in comparison with the shear modulus that represents the variation with both increase and decrease of the scaffold's modulus, due to the increase of the number of pores per face.
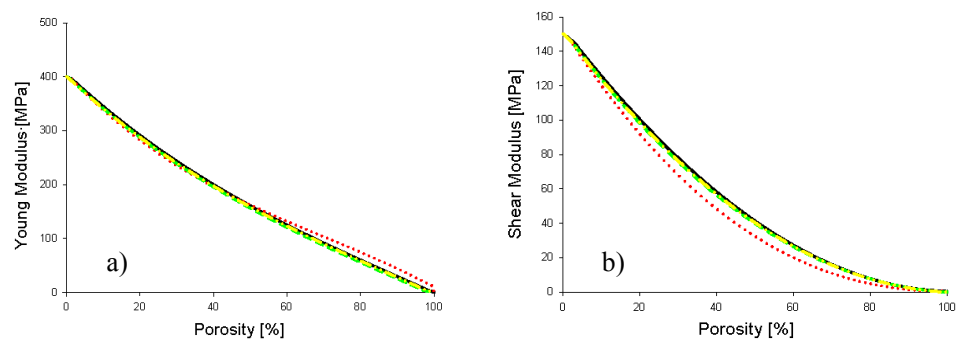

Figure 7: The variation of the material modulus of Poly(caprolactone) material according to the scaffold porosity for all scaffold units a) elastic modulus and b) shear modulus.

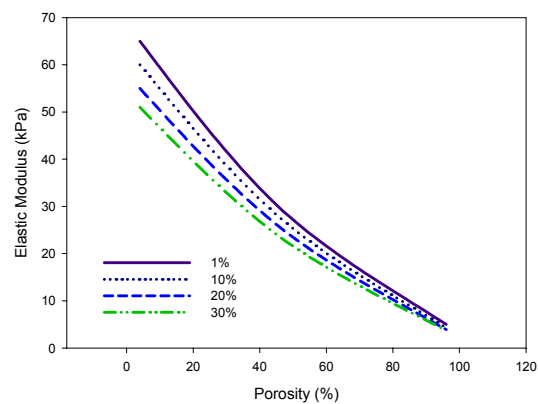

Figure 8: The variation of the elastic modulus versus porosity for circular scaffold units of alginate under different deformation values.
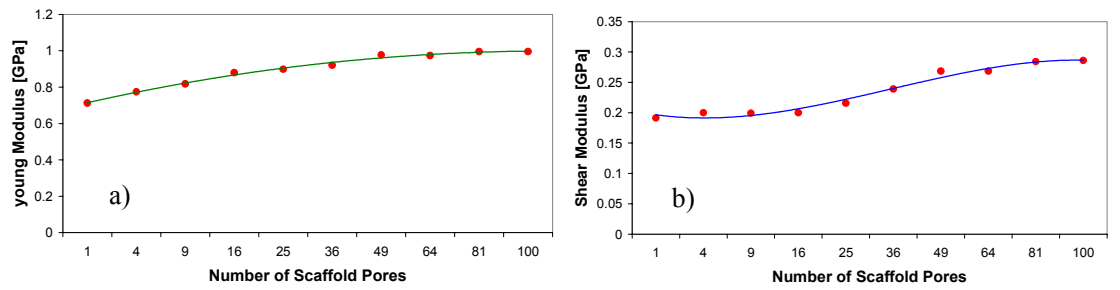

Figure 9: The variation of the material modulus of scaffold units with circular pores in function of the number of pores per face a) elastic modulus and b) shear modulus. 


\section{Biofluid simulations}

To perform the dynamic fluid simulations, certain blood properties have to be defined. At this stage, we intend to understand the flow of blood within the scaffold by only defining the density and the dynamic viscosity. The value introduced for the density value is $1080 \mathrm{Kg} / \mathrm{m}^{3}$ and the dynamic viscosity value is $0.0032 \mathrm{~Pa} \mathrm{~s}$. The inlet value is given as a pressure $(100 \mathrm{~mm} \mathrm{Hg})$. The inlet and outlet of the blood flow within the scaffold is illustrated at Fig. 10.a and Fig. 10.b.
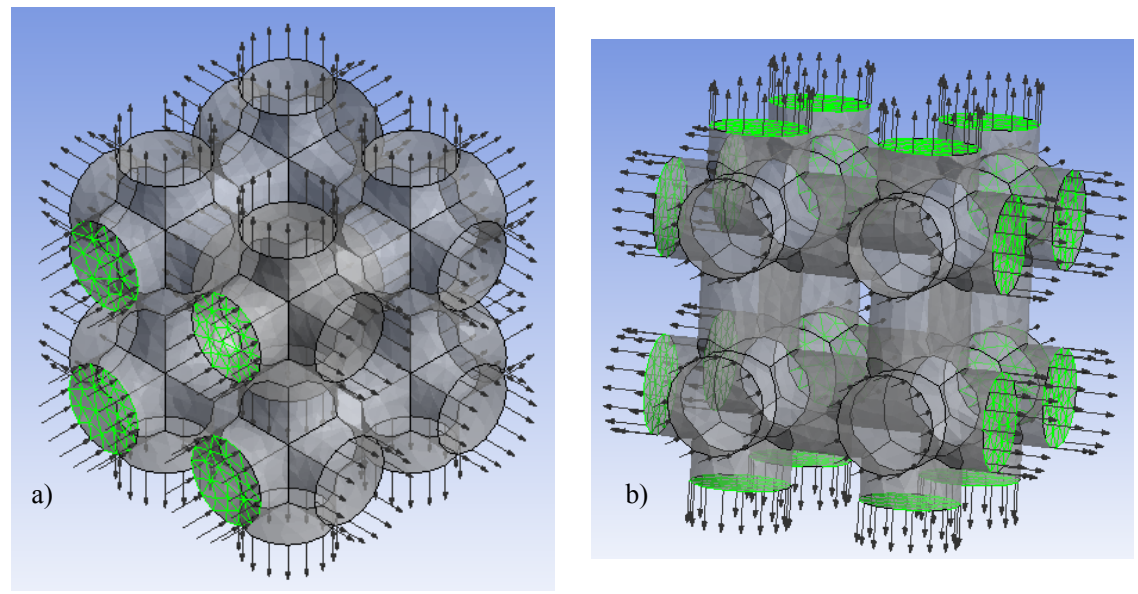

Figure 10: Blood flow within the scaffold a) inlet and b) outlet.

More important results obtained through the simulations are the fluid mass flow, pressure and the fluid velocity. Table 1 lists the mass flow of all 4 scaffolds, being the $4 \mathrm{f}$ pore scaffold unit the highest mass flow value, and the $12 \mathrm{f}$ pore unit the worst value. Excluding the circular pore unit, the mass flow decreases with the increase of the number of faces per pore. The pressure and fluid velocity are illustrated in Fig. 11.a and 11.b. Highest values of pressure and velocity are aligned with the direction of the inlet, indicating that the lateral directions will have less blood flow. Thus, when an equal flow of blood is needed in all directions, the internal scaffold design need to be changed to force an equal blood flow in all directions.

Table 1: $\quad$ Mass flow values for the 4 scaffold units.

\begin{tabular}{|c|c|}
\hline Pore geometry & Mass flow [kg/s] \\
\hline circular & 0.00648326 \\
\hline $4 \mathrm{f}$ & 0.00727725 \\
\hline $8 \mathrm{f}$ & 0.00677977 \\
\hline $12 \mathrm{f}$ & 0.00594014 \\
\hline
\end{tabular}



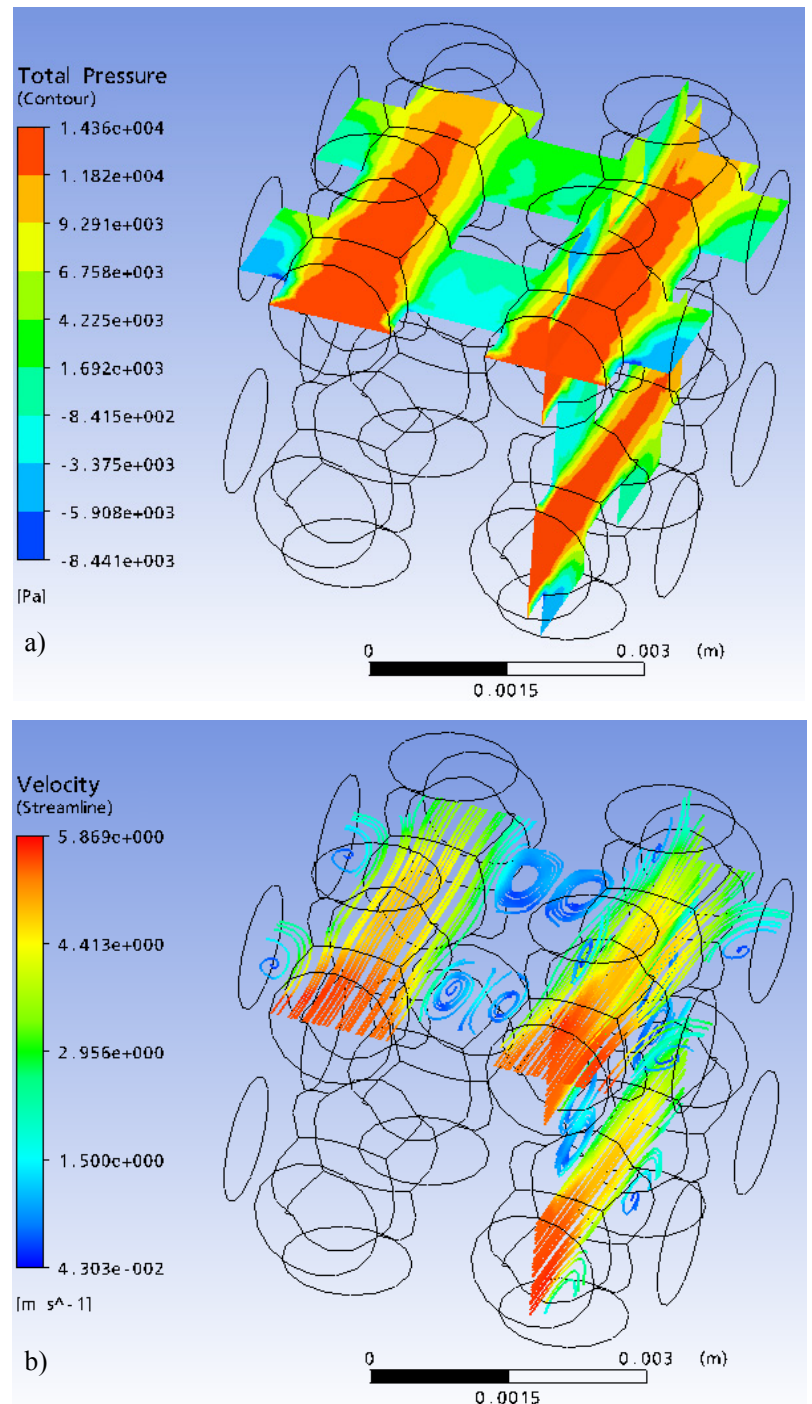

Figure 11: Fluid a) pressure and b) velocity variation within the scaffold.

\section{Conclusions}

Rapid prototyping technologies were recently introduced in the medical field, being particularly viable to produce porous scaffolds for tissue engineering. These scaffolds should be biocompatible, biodegradable, with appropriate porosity, pore structure and pore distribution, on top of having both surface and structural compatibility. Surface compatibility means a chemical, biological and physical suitability with the host tissue. Structural compatibility corresponds to 
the optimal adaptation the mechanical behaviour of the host tissue. Thus, structural compatibility refers to the mechanical properties and deformation capabilities of the scaffold.

This paper presents a computer tool to support the design of scaffolds to be produced by rapid prototyping. The software enables to evaluate scaffold mechanical properties as a function of porosity, for a wide rage of materials being suitable for both hard and soft tissue engineering. Another important aspect is the scaffold's influence regarding the normal flow of biofluids within the human body, which will enable the design of a scaffold offering less resistance to the normal flow of biofluids.

\section{References}

[1] Mendes, A., Lagoa, R. and Bartolo, P.J., Rapid prototyping system for tissue engineering, Proceedings of the International Conference on Advanced Research in Virtual and Physical Prototyping, pp.419-426, 2003.

[2] Network for Organ Sharing website: http://www.unos.org, 2007 March.

[3] L.J. Gibson, Journal of Biomechanics, 2005, 38, 377.

[4] Tsang, V. L., Bhatia, S. N. Three-dimensional tissue fabrication. Advanced Drug Delivery Reviews, 56, 1635-1647, 2004.

[5] M.E. Gomes and R.L. Reis, International Materials Review, 2004, 49, 274.

[6] K.F. Leong, C.M. Cheah and C.K. Chua, Biomaterials, 2003, 24, 2363.

[7] P.J. Bártolo, H. Almeida and T. Laoui, Int J Materials and Product Technology (in press).

[8] J. Reignier and M.A. Huneault, Polymer, 2006, 47, 4703.

[9] P.J. Bártolo, in Design and Nature III: Comparing design in nature with science and engineering, Edited by C.A. Brebbia, WIT Press, Southampton, 2006.

[10] D.W. Hutmacher, M. Sittinger and M.V. Risbud, Trends in Biotechnology, 2004, 22, 7.

[11] C.K. Chua, W.Y. Yeong and K.F. Leong, in Virtual modelling and rapid manufacturing, Edited by P.J. Bártolo et al, Taylor \& Francis, London, 2005 . 